\title{
Ticagrelor Versus Clopidogrel in The Treatment of Elderly Chinese Chronic Total Occlusion Patients Undergoing PCl
}

\section{Peng Han}

981 Hospital of Joint Logistics Support Force

\section{Ying Liang}

Airforce Military Medical University

\section{Suining Xu}

The First Affiliated Hospital of Xi'an Medical University

\section{Shuai Zhao}

Xijing Hospital, Airforce Military Medical University

\section{Yan Chen}

971 Hospital of the PLA Navy

\section{Ziwei Wang}

Airforce Military Medical University

\section{Yuhao Chen}

Airforce Military Medical University

\section{Boda Zhu}

Airforce Military Medical University

\section{Zhilin Sha}

Airforce Military Medical University

\section{Anxin Shen}

Airforce Military Medical University

\section{Feng Tao}

Naval Medical University

\section{Qin Wang}

Airforce Military Medical University

\section{Qiong Wang}

Xijing Hospital, Airforce Military Medical University

\section{Genrui Chen}

Hanyin County People's Hospital

\section{Zhijun Tan}

Airforce Military Medical University

Li Yang 
Xijing Hospital, Airforce Military Medical University

\section{Haokao Gao}

Xijing Hospital, Airforce Military Medical University

\section{Chengxiang Li}

Xijing Hospital, Airforce Military Medical University

Kun Lian ( $D$ michealo@qq.com )

Xijing Hospital, Airforce Military Medical University

\section{Research Article}

Keywords: Ticagrelor, Clopidogrel, aged, percutaneous coronary intervention, chronic total occlusion

Posted Date: January 7th, 2022

DOI: https://doi.org/10.21203/rs.3.rs-1195001/v1

License: (1) (i) This work is licensed under a Creative Commons Attribution 4.0 International License. Read Full License 


\section{Abstract}

Background: Taking thrombosis and bleeding risks into consideration, little real world study data is available to dual antiplatelet therapy (DAPT) after percutaneous coronary intervention (PCl) in elderly Chinese chronic total occlusion (CTO) patients.

Methods: We assigned 504 CTO patients aged $\geq 75$ years who successfully underwent $\mathrm{PCl}$ from December 2009 to May 2020. The patients were randomized to Clopidogrel and Ticagrelor group and received DAPT for up to 12 months. Efficacy endpoints were evaluated by major adverse cardiac events (MACE) consisting of all-cause death, nonfatal myocardial infarction (MI) and clinically driven revascularization. The safety endpoints were recorded as the incidence of Bleeding Academic Research Consortium (BARC) bleeding.

Results: Patients in Clopidogrel group were older, they had a higher percentage of BMI, diastolic blood pressure and HDL-C than those in Ticagrelor group. Clopidogrel group had a lower percentage of hyperlipidemia, prior PCl, glucose, TG and LDL-C. No significant difference was found as to the Angiographic and procedural characteristics (P凶0.05 for all). After 12 months' follow-up, the incidence of MACE $(12.19 \%$ vs. $11.04 \%, P=0.763)$ and bleeding $(9.38 \%$ vs. $13.64 \%, P=0.205)$ had no significant difference. After clinical characteristics balanced matching by IPTWs model, we found that Ticagrelor had an unfavorable effect on reducing the incidence of bleeding with the IPTWs model (IPTW-OR, 1.81, 95\% Cl: 1.18-2.76, $\mathrm{P}=0.006)$.

Conclusions: This clinical study demonstrated that Clopidogrel should be recommended to elderly CTO patients after $\mathrm{PCl}$, especially those with a high bleeding risk.

Trial registration: The study protocol was approved by the Ethics Committee of Air Force Medical University (KY20172019-1).

\section{Background}

Coronary CTO is observed in approximatively $15-25 \%$ of CAD patients have undergone prior coronary angiography ${ }^{[1,2]}$. Guidelines recommend selective $\mathrm{PCl}$ to $\mathrm{CTO}$ patients to improve their symptoms and quality of life, and patients who undergo successful CTO-PCI have higher survival rates ${ }^{[3,4]}$. As CTO lesions are complex and intraoperative stent placement is frequent, the risk of thrombosis is high; therefore, intensive DAPT is recommended for CTO-PCl patients to prevent stent thrombosis ${ }^{[5,6]}$. However, continuous antiplatelet therapy is also associated with increased bleeding risk, leading to the necessity to balance the risk of bleeding and ischemia. As the elderly population over 75 years has a much higher risk of bleeding, more concern is needed for the adjustment of DAPT medication to elderly CTO-PCI patients. Consequently, it is of great significance to investigate the efficacy and safety of different DAPT strategies for elderly CTO-PCI patients. However, traditional clinical randomized controlled trials seldom focused on elderly CTO patients, and data on Chinese elderly CTO patients after PCI is scarcely available. In addition, most CTO-PCI DAPT studies focused on duration rather than medication 
selection, which makes current clinical studies of Chinese elderly CTO-PCI patients' DAPT medication selection underrepresented.

Ticagrelor and Clopidogrel are two of the most commonly used DAPT drugs. Compared with Clopidogrel, Ticagrelor has faster action, stronger antiplatelet effect and its effect is reversible ${ }^{[7]}$. A study on Chinese CTO-PCI patients investigated the efficacy and safety of Ticagrelor and Clopidogrel, in which there were three groups (Ticagrelor group of $90 \mathrm{mg}$ twice daily, Ticagrelor group of $60 \mathrm{mg}$ twice daily, and Clopidogrel group of $75 \mathrm{mg}$ once daily). The major adverse cardiac and cerebral events (MACCE, all-cause mortality, target vessel revascularization, stent thrombosis, nonfatal $\mathrm{MI}$, and nonfatal stroke), major bleeding and minor bleeding incidence were separately $7.3 \%$ vs. $6.4 \%$ vs. $14.2 \%,(P=0.023), 4.1 \%$ vs. $0.6 \%$ vs. $0.6 \%,(P=0.016)$ and $23.4 \%$ vs. $12.4 \%$ vs. $11.9 \%,(P=0.004)^{[8]}$. These results indicate that Ticagrelor with normal dose has lower incidence of MACE but higher bleeding incidence compared with Clopidogrel in Chinese CTO-PCl patients. However, the study did not reveal information on elderly patients, and there is no study related to elderly CTO patients' DAPT. Therefore, it of necessity to investigate elderly CTO-PCI patients.

Herein, the current study is designed to assess the efficacy and safety of Ticagrelor in comparison with Clopidogrel on a background of Aspirin for elderly Chinese CTO patients who have undergone prior PCI.

\section{Methods}

\section{Study design}

This study was conducted in the department of cardiology, Xijing Hospital from December 2009 to May 2020 to compare the efficacy and safety of Ticagrelor versus Clopidogrel in elderly Chinese CTO patients who previously underwent elective $\mathrm{PCl}$ with drug-eluting stents (DES). PCl success was assessed by the interventional cardiologist performing the procedure. The study protocol was approved by the Ethics Committee of Air Force Medical University (KY20172019-1). Written informed consents were obtained from all participants.

\section{Study participants}

From December 2009 to May 2020, a total of 504 CTO patients who successfully underwent elective PCl followed by DAPT for up to 12 months were consecutively enrolled in the study and randomized to treatment with Ticagrelor or Clopidogrel. Of the 504 patients, 30 were lost to follow-up, and eventually 474 patients were included in this study. For these participants, 320 of them took $100 \mathrm{mg}$ Aspirin and Clopidogrel with a $300 \mathrm{mg}$ loading dose followed by a dose of $75 \mathrm{mg}$ daily, while the other 154 patients took $100 \mathrm{mg}$ Aspirin and Ticagrelor with a loading dose of $180 \mathrm{mg}$ followed by a dose of $90 \mathrm{mg}$ twice daily. All the patients took Aspirin at a dose of $100 \mathrm{mg}$ daily. СTO was defined as angiographic evidence of total occlusion with complete interruption of anterograde blood flow (Thrombolysis In Myocardial Infarction (TIMI) flow grade 0 ) with an estimated duration of $>3$ months via previous angiograms, angina symptoms and a history of $\mathrm{Ml}^{[2]}$. Coronary arteries measured were proximal left main artery (LM), left 
anterior descending artery (LAD), right coronary artery (RCA) and left circumflex artery (LCX). Inclusion criteria included: 1 ) age $\geq 75$ years old; 2 ) confirmed with CTO by coronary angiography; 3 ) successful $\mathrm{PCl} ; 4)$ informed consent signed by the patient. Exclusion criteria included: 1) conservative oral anticoagulation therapy; 2) PCl contraindications; 3) P2Y12 inhibitors contraindications; 4) high risk of bleeding diathesis or coagulation disorder; 5) dialysis-dependent renal failure or liver cirrhosis; 6) refusal to participate in this study by the patient. Clinical, angiographic and procedural baseline data were collected and recorded.

\section{Study end points}

Clinical follow-up was carried out by telephone interviews and outpatient visits. The follow-up period started from the date of DAPT use after PCl and ended when any study outcome first occurred or at 12 months after PCl. Inpatient observation and outpatient visits were scheduled for patients being regularly followed up in our hospital, while telephone calls were made for patients without regular medical followup. The incidence of end points was collected in medical records by a predefined questionnaire, in which health status, physical examinations, vital signs as well as laboratory assessments were simultaneously recorded. The efficacy endpoints in this study were evaluated by the occurrence of MACE, i.e., the composite of all-cause death, nonfatal $\mathrm{Ml}$ and clinically driven revascularization. All-cause death was defined as death from any cause, which was ascertained without adjudication ${ }^{[9]}$. MI was defined as the presence of recurrent cardiac ischemic symptoms, new $Q$ waves in $\geq 2$ contiguous electrocardiographic leads or an elevation of creatine kinase (CK) level or its MB isoenzyme (CK-MB) to at least 3 times the upper limit of normal in 2 plasma samples ${ }^{[10]}$. Clinically driven revascularization was defined as any reintervention because of symptoms ${ }^{[11]}$. The safety endpoints were evaluated by the incidence of bleeding: Bleeding Academic Research Consortium (BARC) type 1, 2, 3, or $5^{[12]}$. Type 1 is inactive bleeding. Type 2 is active bleeding requiring evaluation or intervention by medical personnel, which differs from Type 3, Type 5 and bleeding related to coronary artery bypass graft. Type 3 is heavy bleeding as well as intracranial bleeding with significant hemoglobin reduction to $5 \mathrm{~g} / \mathrm{dl}$, which requires blood transfusion. For Type 5, it refers to potential or qualitative fatal bleeding. The major bleeding events, which are the equivalent of BARC 3 and 5 , include gastrointestinal bleeding, intracranial hemorrhage, hemoglobin decrease of $\geq 3 \mathrm{~g} / \mathrm{dL}$, significant bleeding requiring blood transfusion, and fatal bleeding ${ }^{[13]}$.

\section{Statistical analysis}

Continuous variable was described as mean \pm SD or median and interquartile spacing, and categorical variable as number (percentage). Differences in continuous and categorical variables between groups were analyzed with Mann-Whitney U-test and Chi-square test respectively, and $P<0.05$ was considered to be statistically significant. Univariate logistic regression models were developed to explore the effect of treatment. To validate the effects of treatment groups on the incidence of bleeding, a propensity score weighting method was adopted according to the results of univariate factor comparison and literature reports. A logistic model was used to calculate propensity score, in which the dependent variable was Ticagrelor group and the covariates included age, DBP, hyperlipidemia, prior PCl, GLU, TG, LDL-C and HDLC. Standardized mean differences (SMD) $(<0.20$ is indicative of good balance) were calculated to 
evaluate the balance of the inverse probability of treatment weighting (IPTW) model. Finally, the IPTW odds ratio (IPTW-OR) was derived for Ticagrelor group.

\section{Results}

\section{Clinical, angiographic and procedural baseline characteristics}

In this study, a total of 504 CTO patients ( $\geq 75$ years) were prescribed with DAPT for 12 months after PCl from December 2009 to May 2020. During this period of time (12 months after PCl), 30 patients were lost to follow-up, and in the end 474 СTO patients were included in the study (Figure 1). In terms of clinical baseline characteristics, compared with the patients in Ticagrelor group, those in Clopidogrel group were older ( $80.45 \pm 4.23$ vs. $79.18 \pm 3.59, P=0.001)$ and had a higher percentage of BMI $(24.14 \pm 3.49$ vs.

$22.82 \pm 4.75, P=0.003)$, more elevated diastolic blood pressure ( $74.53 \pm 10.16$ vs. $71.82 \pm 9.85, P=0.007)$ and higher HDL-C (1.92 \pm 0.87 vs. $1.05 \pm 0.29, P<0.001)$. A lower percentage of patients in Clopidogrel group had hyperlipidemia ( $22.50 \%$ vs. $43.51 \%, P<0.001)$ and prior $\mathrm{PCl}(25.63 \%$ vs. $39.61 \%, P=0.003)$, and they had lower glucose ( $24.14 \pm 3.49$ vs. $22.82 \pm 4.75, P=0.003)$, TG (1.31 \pm 0.78 vs. $1.48 \pm 0.85, P=0.037)$ and LDL-C $(1.22 \pm 0.71$ vs. $1.96 \pm 0.75, P<0.001)$. There were no significant differences among the other clinical characteristics ( $P>0.05$ for all) (Table 1). For CTO lesion characteristics, no significant differences were found as to the CTO lesions of coronary arteries (LM, LAD, RCA, and LCX), number of treated vessels, number of stents or total stent length ( $P>0.05$ for all) (Table 2 ). 
Table 1

Baseline clinical characteristics $(n=474)$

\begin{tabular}{|c|c|c|c|}
\hline Variable & Clopidogrel $(n=320)$ & Ticagrelor $(n=154)$ & $P$ value \\
\hline Age, years & $80.45 \pm 4.23$ & $79.18 \pm 3.59$ & 0.001 \\
\hline Male, \% & $230(71.88)$ & $115(74.68)$ & 0.582 \\
\hline BMI & $24.14 \pm 3.49$ & $22.82 \pm 4.75$ & 0.003 \\
\hline Heart rate, beats/min & $73.15 \pm 12.33$ & $72.24 \pm 10.74$ & 0.437 \\
\hline SBP, mmHg & $130.02 \pm 22.71$ & $128.39 \pm 25.38$ & 0.487 \\
\hline DBP, mmHg & $74.53 \pm 10.16$ & $71.82 \pm 9.85$ & 0.007 \\
\hline Smoking, \% & $87(27.19)$ & $33(21.43)$ & 0.215 \\
\hline \multicolumn{4}{|l|}{ Medical history } \\
\hline Hypertension, \% & $192(60.00)$ & $85(55.19)$ & 0.322 \\
\hline Diabetes mellitus, \% & $90(28.13)$ & 48 (31.17) & 0.518 \\
\hline Hyperlipidemia & $72(22.50)$ & $67(43.51)$ & $<0.001$ \\
\hline Valvular heart disease, \% & $2(0.63)$ & 0 & 1.000 \\
\hline Atrial fibrillation, \% & $12(375)$ & $4(2.60)$ & 0.598 \\
\hline Stroke, \% & $33(10.31)$ & 19 (12.34) & 0.532 \\
\hline Chronic kidney diseases, \% & $8(2.50)$ & $8(5.19)$ & 0.172 \\
\hline Peripheral arterial disease, $\%$ & $2(0.63)$ & 0 & 1.000 \\
\hline Family history of CAD, \% & 0 & $2(1.30)$ & 0.105 \\
\hline Prior MI, \% & 15 (4.69) & $10(6.49)$ & 0.511 \\
\hline Prior PCl, \% & $82(25.63)$ & $61(39.61)$ & 0.003 \\
\hline Prior CABG, \% & $8(2.50)$ & $2(1.30)$ & 0.511 \\
\hline \multicolumn{4}{|l|}{ Laboratory data } \\
\hline WBC $\left(10^{9} / \mathrm{L}\right)$ & $6.65 \pm 2.19$ & $6.87 \pm 3.79$ & 0.420 \\
\hline
\end{tabular}

Abbreviations: $\mathrm{CAD}$, coronary artery disease; $\mathrm{Ml}$, myocardial infarction; $\mathrm{PCl}$, percutaneous coronary intervention; CABG, coronary artery bypass grafting; BMI, Body Mass Index; SBP, systolic blood pressure; DBP, diastolic blood pressure; WBC, white blood cell; RBC, red blood cell; $\mathrm{Hb}$, Hemoglobin; PLT, blood platelet ; Ccr, creatinine clearance; BUN, blood urea nitrogen; UA, uric acid; ALT, alanine amiotransferase; AST, aspartate aminotransferase; Alb, albumin; Glu, Glucose; TC, triglyceride; TG, total cholesterol; LDL-C, low-density lipoprotein cholesterol; HDL-C, high-density lipoprotein cholesterol; LVEF, left ventricular ejection fraction. 


\begin{tabular}{|c|c|c|c|}
\hline Variable & Clopidogrel $(n=320)$ & Ticagrelor $(n=154)$ & $P$ value \\
\hline $\operatorname{RBC}\left(10^{12} / \mathrm{L}\right)$ & $4.24 \pm 0.61$ & $4.24 \pm 0.53$ & 0.946 \\
\hline $\mathrm{Hb}(\mathrm{g} / \mathrm{L})$ & $131.19 \pm 18.45$ & $129.87 \pm 17.01$ & 0.457 \\
\hline PLT $\left(10^{9} / \mathrm{L}\right)$ & $177.32 \pm 67.05$ & $189.04 \pm 60.24$ & 0.066 \\
\hline Creatinine $(\mu \mathrm{mol} / \mathrm{L})$ & $108.20 \pm 52.01$ & $114.93 \pm 82.86$ & 0.288 \\
\hline $\operatorname{Ccr}(\mathrm{ml} / \mathrm{min})$ & $49.09 \pm 13.88$ & $46.40 \pm 13.54$ & 0.104 \\
\hline BUN (mmol/L) & $5.89 \pm 2.20$ & $6.06 \pm 1.97$ & 0.655 \\
\hline $\mathrm{UA}(\mu \mathrm{mol} / \mathrm{L})$ & $311.00 \pm 289.95$ & $313.27 \pm 89.20$ & 0.930 \\
\hline $\operatorname{ALT}(\mathrm{U} / \mathrm{L})$ & 20.00 (16.75) & $21.00(11.00)$ & 0.578 \\
\hline AST (U/L) & $20.00(13.00)$ & $16.00(12.85)$ & 0.177 \\
\hline Alb (g/L) & $38.49 \pm 4.99$ & $37.96 \pm 3.94$ & 0.363 \\
\hline Glu (mmol/L) & $6.19 \pm 2.42$ & $6.97 \pm 3.04$ & 0.007 \\
\hline TC (mmol/L) & $3.64 \pm 1.07$ & $3.52 \pm 0.89$ & 0.236 \\
\hline TG (mmol/L) & $1.31 \pm 0.78$ & $1.48 \pm 0.85$ & 0.037 \\
\hline LDL-C(mmol/L) & $1.22 \pm 0.71$ & $1.96 \pm 0.75$ & $<0.001$ \\
\hline $\mathrm{HDL}-\mathrm{C}(\mathrm{mmol} / \mathrm{L})$ & $1.92 \pm 0.87$ & $1.05 \pm 0.29$ & $<0.001$ \\
\hline NT-proBNP (pg/ml) & 598.30 (1415.98) & 661.50 (2065.50) & 0.229 \\
\hline LVEF (\%) & $52.17 \pm 9.36$ & $51.12 \pm 9.29$ & 0.303 \\
\hline \multicolumn{4}{|c|}{$\begin{array}{l}\text { Abbreviations: CAD, coronary artery disease; MI, myocardial infarction; PCI, percutaneous coronary } \\
\text { intervention; CABG, coronary artery bypass grafting; BMI, Body Mass Index; SBP, systolic blood } \\
\text { pressure; DBP, diastolic blood pressure; WBC, white blood cell; RBC, red blood cell; Hb, Hemoglobin; } \\
\text { PLT, blood platelet ; Ccr, creatinine clearance; BUN, blood urea nitrogen; UA, uric acid; ALT, alanine } \\
\text { amiotransferase; AST, aspartate aminotransferase; Alb, albumin; Glu, Glucose; TC, triglyceride; TG, } \\
\text { total cholesterol; LDL-C, low-density lipoprotein cholesterol; HDL-C, high-density lipoprotein cholesterol } \\
\text { LVEF, left ventricular ejection fraction. }\end{array}$} \\
\hline
\end{tabular}


Table 2

Angiographic and procedural characteristics

\begin{tabular}{llll}
\hline Variable & Clopidogrel $(\mathbf{n}=\mathbf{3 2 0})$ & Ticagrelor $(\mathbf{n = 1 5 4})$ & $P$ value \\
\hline CTO Lesion characteristics, \% & & & \\
\hline LM & $2(0.63)$ & 0 & 1.000 \\
\hline RCA & $149(46.56)$ & $76(49.35)$ & 0.624 \\
\hline LAD & $158(49.38)$ & $71(46.10)$ & 0.556 \\
\hline LCX & $96(30.00)$ & $53(34.42)$ & 0.343 \\
\hline Number of treated CTO vessels, \% & & & 0.242 \\
\hline 1 & $308(96.25)$ & $144(93.51)$ & 0.342 \\
\hline 2 & $12(3.75)$ & $9(5.84)$ & 0.325 \\
\hline 3 & 0 & $1(0.65)$ & 0.842 \\
\hline Number of stents & $2.42 \pm 1.16$ & $2.40 \pm 1.15$ & 0.948 \\
\hline Total stent length, mm & $61.13 \pm 34.18$ & $61.36 \pm 36.55$ & \\
\hline
\end{tabular}

Abbreviations: CTO, chronic total occlusion; LM, left main; LAD, left anterior descending; RCA, right coronary artery; LCX, left circumflex.

\section{Clinical outcomes on follow-up}

After 12 months' follow-up, the incidence of MACE was $12.19 \%$ in Clopidogrel group and $11.04 \%$ in Ticagrelor group, with no statistical significance ( $P>0.05)$. The individual components in the two groups were not significantly different either, which included all-cause death (10.94\% vs. $11.04 \%)$, nonfatal MI $(0.31 \%$ vs. $1.30 \%)$ and clinically driven revascularization $(0.94 \%$ vs. 0$)$. The total bleeding rate $(9.38 \%$ vs. $13.64 \%$ ) and BARC 1 bleeding (8.13\% vs. $12.99 \%$ ) of Clopidogrel group were lower than those of Ticagrelor group, but with no statistical significance ( $P>0.05)$ (Table 3$)$. Considering that the factors that might be related to bleeding were not balanced, the IPTW model was used to balance the clinical characteristics from the two groups (Figure 2). The characteristics were significantly balanced after matching, and it was found that compared with Clopidogrel, Ticagrelor had an adverse impact on the reduction of the incidence of bleeding with the IPTW model (IPTW-OR: 1.81, 95\% Cl: 1.18-2.76, $P=0.006$ ) (Figure 3). 
Table 3

Efficacy and safety points

\begin{tabular}{|llll|}
\hline Variable & Clopidogrel $(\mathrm{n}=320)$ & Ticagrelor $(\mathrm{n}=154)$ & $\boldsymbol{P}$ value \\
\hline MACE, \% & $39(12.19)$ & $17(11.04)$ & 0.763 \\
\hline All-cause death, \% & $35(10.94)$ & $17(11.04)$ & 1.000 \\
\hline Nonfatal myocardial infarction, \% & $1(0.31)$ & $2(1.30)$ & 0.248 \\
\hline Clinically driven revascularization, \% & $3(0.94)$ & 0 & 0.554 \\
\hline Bleeding, \% & $30(9.38)$ & $21(13.64)$ & 0.205 \\
\hline BARC 1,\% & $26(8.13)$ & $20(12.99)$ & 0.100 \\
\hline BARC 2, \% & $2(0.63)$ & 0 & 1.000 \\
\hline BARC 3, \% & 0 & 0 & 1.000 \\
\hline BARC 5, \% & $2(0.63)$ & $1(0.65)$ & \\
\hline
\end{tabular}

\section{Discussion}

$\mathrm{PCl}$ is feasible for CTO with high success rates ${ }^{[14]}$, but CTO-PCI patients have higher ischemic risk because of more complex lesions. Therefore, prolonged DAPT duration is recommended for these patients ${ }^{[2,5]}$. Elderly CTO-PCI patients are also at high risk of bleeding ${ }^{[15]}$, which makes it vitally important to balance the risks of both thrombose and hemorrhage for CTO-PCI patients to ensure a desirable prognosis. Previous studies indicated that the improvement of clinical outcomes was irrespective of whether the duration of DAPT after PCl in CTO patients was $>12$ months or not $[16,17]$. Considering the reasons above, the duration of DAPT in this study was determined as 12 months. DAPT, which comprises aspirin and a P2Y12 inhibitor, prevents both stent thrombosis and non-culprit segments thrombosis via antiplatelet aggregation to reduce coronary ischemic events after PCl. As P2Y12 inhibitors recommended by many guidelines, Ticagrelor and Clopidogrel can prevent adenosine diphosphate (ADP) dependent activation of platelet aggregation by binding to the $\mathrm{P} 2 \mathrm{Y} 12$ receptor ${ }^{[5,6]}$.

According to the results of the PLATO trial, compared with Clopidogrel, Ticagrelor showed a lower incidence of endpoint events including cardiovascular death, $\mathrm{Ml}$ and stroke $(9.8 \%$ vs. $11.7 \%, P<0.001)$ without increasing bleeding risk $(11.6 \% \text { vs. } 11.2 \%, P=0.43)^{[18,19]}$. In the ESTATE study that enrolled Taiwan acute coronary syndrome (ACS) patients, compared with patients taking Clopidogrel, those who took Ticagrelor had a lower incidence of $\mathrm{Ml}$, stroke, or vascular death endpoints with marginal statistical significance ( $7.1 \%$ vs. $11.6 \%, P=0.07)$, and the incidence of all bleeding was similar $(19.6 \%$ vs. $14.3 \%$, $P=0.13)^{[20]}$. Among these studies, more than $80 \%$ patients received invasive therapies ( $\mathrm{PCl}$ or coronary artery bypass grafting (CABG)). The PLATO trial contained 587 Chinese patients (3.1\%) and also 2878 elderly patients $(15.45 \%){ }^{[18,19]}$, and the ESTATE study included $269(28.99 \%)$ Chinese patients older than 
75 years ${ }^{[20]}$. In these studies data concerning СTO patients was absent, and Chinese elderly patients accounted only for a small number of the subjects enrolled in the studies. Consequently, these studies could hardly be representative of Chinese elderly CTO patients.

In the present study, all the patients were aged $\geq 75$ years and underwent $\mathrm{PCl}$ with DES. After 12-month DAPT, the incidence of MACE in Ticagrelor group was lower than that in Clopidogrel group $(11.04 \% \mathrm{vs}$. $12.19 \%)$, but the incidences of overall bleeding (13.64\% vs. $9.38 \%)$ and BARC 1 bleeding $(12.99 \%$ vs. 8.13\%) were higher in Ticagrelor group, with no statistical significance for all the differences. After balancing clinical characteristics of the two groups, the difference of bleeding incidence was found to be statistically significant. This indicates that Ticagrelor has similar effect in reducing MACE but higher bleeding risk for Chinese elderly CTO-PCI patients. Thus, Ticagrelor showed similar efficiency but worse safety in comparison with Clopidogrel in our study.

Several studies showed that Clopidogrel had similar MACE but higher bleeding incidence than Ticagrelor for patients after $\mathrm{PCl}{ }^{[21-23]}$. There is a "East Asian paradox" which describes that East Asian patients have lower ischemic but higher bleeding risk after $\mathrm{PCI}{ }^{[24]}$. In the PHILO trial which targeted mostly Japanese patients, compared with Clopidogrel, Ticagrelor was associated with higher incidence of overall bleeding events (23.8\% vs. $14.7 \%$, hazard ratio (HR): $1.72 ; 95 \% \mathrm{Cl}: 1.23-2.40)$ and minor bleeding events (15.2\% vs. $9.2 \%$, HR: $1.75 ; 95 \% \mathrm{Cl}: 1.15-2.67)$, and the incidence of ischemic events (the composite of MI, stroke or vascular-cause death) is not significantly different (9.0\% vs. $6.3 \%$, HR: $1.47 ; 95 \%$ Cl: $0.88-2.44)$ [25]. The TICAKOREA trial indicated that in comparison with Clopidogrel group, Ticagrelor group had higher incidence of clinically significant bleeding $(11.7 \%$ vs. $5.3 \%, P=0.002)$ and minor bleeding $(5.2 \%$ vs. $1.3 \%, P=0.02)$, and the incidence of cardiovascular death, $\mathrm{Ml}$ and stroke was not significantly different between the two groups $(9.2 \% \text { vs. } 5.8 \%, P=0.07)^{[26]}$. The Kamir-NIH study based on East Asian population showed that Ticagrelor reduced the risk of ischemic event with statistical significance (8.6\% vs. $11.9 \%$, $P=0.018)$, but it had a significantly higher bleeding risk than Clopidogrel $(10.8 \%$ vs. $4.8 \%, P<0.001)$ for patients with acute myocardial infarction (AMI) and multivessel disease (MVD) ${ }^{[27]}$. These studies support the perception that East Asian patients with the medication of Ticagrelor have a higher incidence of bleeding complications, especially minor bleeding, which is consistent with our findings.

There are few studies aiming at DATP for Chinese CTO patients or elderly patients who underwent PCl, let alone post- $\mathrm{PCl}$ patients with CTO who aged $\geq 75$ years in China. In a study on Chinese CTO patients who underwent $\mathrm{PCl}$, the incidences of overall MACE, major bleeding and minor bleeding in normal Ticagrelor dose group and Clopidogrel group were (7.3\% vs. $14.2 \%),(4.1 \%$ vs. $0.6 \%)$ and $(23.4 \%$ vs. $11.9 \%)$, respectively ${ }^{[8]}$. This indicates that normal dose Ticagrelor renders lower incidence of MACE but higher bleeding incidence compared with Clopidogrel for Chinese CTO patients. According to the POPular AGE study involving 1002 patients (aged $\geq 70$ years) with non-ST-elevation acute coronary syndrome (NSTEACS), the primary bleeding outcome incidence was higher in Ticagrelor group ( $24 \%$ vs. $18 \%, P=0.02)$, and there were no significant differences as to the incidence of cardiovascular death, $\mathrm{Ml}$, and stroke between 
the two groups (11\% vs. $12 \%, P=0.71)^{[28]}$, which is in favor of the conclusion that Clopidogrel is recommended for elderly NSTE-ACS patients with a high bleeding risk.

The MACE incidence in the POPular AGE study is similar to that in our study, whereas in other studies the MACE incidence of Ticagrelor group is lower than that in our study. This might be attributed to the greater complexity of lesions of elderly CTO patients, which leads to higher MACE risks. The bleeding incidence in Ticagrelor group in our study is higher than those in the TICAKOREA trial and Kamir-NIH study but is lower than incidences in other studies. This may be partly due to the research bias brought by small sample size as well as the differences of baseline and procedure characteristics between the groups that might affect the comparison of the endpoints.

It is found that DAPT after PCl can benefit elderly CTO-PCI patients, and Ticagrelor showed similar MACE and higher incidence of bleeding, especially minor bleeding (BARC 1 bleeding) compared with Clopidogrel; therefore, we believe Clopidogrel has similar efficiency and better safety for elderly Chinese CTO-PCI patients. As Ticagrelor has higher bleeding incidence, it should be prescribed with caution to patients with a high bleeding risk. This study may make potential contribution to clinical practice, but it is essential to carry out a further prospective, multi-center and large-scale study to compare the efficacy and safety outcomes of Ticagrelor and Clopidogrel in elderly Chinese CTO patients who underwent PCI.

\section{Study limitations}

There are several limitations in the current study. Firstly, it was a single center study with a small sample size, in which the selection bias was hardly avoidable. Secondly, we did not distinguish cardiovascular death in our follow-up, and we did not assess CTO score, opening techniques or the occurrence of stroke. Thirdly, owing to the unavailability of the information on the date of nonfatal $\mathrm{Ml}$ and clinically driven revascularization, we did not adjust the analysis of efficacy end points accordingly. Fourthly, we did not include other related therapeutic agents such as $\beta$-blocker and other drugs, and we did not evaluate the major adverse effects of P2Y12 inhibitors including dyspnea, hyperuricemia, and asymptomatic heart block.

\section{Conclusions}

This clinical study demonstrated that in the improvement of elderly Chinese CTO patients' prognosis, Clopidogrel is found to be more desirable for elderly Chinese CTO patients after PCl because of lower incidence of bleeding events. Consequently, Clopidogrel should be recommended for elderly CTO patients after $\mathrm{PCl}$, especially patients with a high bleeding risk.

\section{Abbreviations}

DAPT: dual antiplatelet therapy; PCl: percutaneous coronary intervention; CTO: chronic total occlusion; MACE: major adverse cardiac event; MI: myocardial infarction; BARC: Bleeding Academic Research

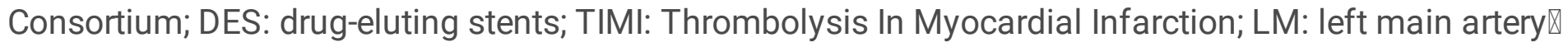


LAD: left anterior descending artery; RCA: right coronary artery; LCX: left circumflex artery; CK: creatine kinase; CK-MB: creatine kinase MB isoenzyme; SMD: standardized mean differences; IPTW: inverse probability of treatment weighting; ADP: adenosine diphosphate; ACS: acute coronary syndrome; CABG: coronary artery bypass grafting; AMI: acute myocardial infarction; MVD: multivessel disease; NSTE-ACS: non-ST-elevation acute coronary syndrome.

\section{Declarations}

\section{Ethics approval and consent to participate}

The experimental protocol was established, according to the ethical guidelines of the Helsinki Declaration and was approved by the Human Ethics Committee of Air Force Medical University (KY20172019-1). Written informed consents were obtained from all participants.

\section{Consent for publication}

The consent was obtained from all authors for publication of this study.

\section{Availability of data and materials}

The datasets generated and analysed during the current study are available from the corresponding author on reasonable request.

\section{Funding}

This work was supported by Shaanxi province Key Research and Development Program General ProjectSocial Development (2018SF-153), Xi'an Science and Technology Project (20YXYJ0003(4)), New Clinical Technology and New Business of Xijing Hospital (XJGX15Y39), Bethune-Merck Diabetes Research Foundation (G2017044).

\section{Conflict of interest statement}

The authors have no conflicts of interest to declare.

\section{Authors' contributions}

Peng Han contributed to the original draft writing and editing of the work; Suining Xu contributed to the design and editing; Ying Liang and Zhijun Tan contributed to the statistical analyses; Shuai Zhao, Yan Chen, Ziwei Wang, Yuhao Chen, Boda Zhu, Zhilin Sha and Anxin Shen contributed to the data curation; Feng Tao contributed to the language editing; Qin Wang, Qiong Wang, Genrui Chen, Li Yang and Haokao Gao contributed to the review and editing; Kun Lian and Chengxiang Li contributed to the project administration and supervision. All authors read and approved the final manuscript.

\section{Acknowledgements}


The authors are grateful to Qing Yan, Jianan Wu and Jin Li for their help with the collection of data in this study.

\section{Authors' information}

Peng Han, Ying Liang, and Suining Xu contributed equally to this work.

Corresponding author:

Kun Lian

Department of Cardiology, Xijing Hospital, Airforce Military Medical University, 169 West Changle Road, Xi'an, Shaanxi 710032, P.R. China

Telephone: 0086-17792398286

E-mail:michealo@qq.com.

Co-corresponding author:

Chengxiang Li

Department of Cardiology, Xijing Hospital, Airforce Military Medical University, 169 West Changle Road, Xi'an, Shaanxi 710032, P.R. China

Telephone: 0086-13992816228

E-mail: lichx1@163.com

\section{Author details}

${ }^{1}$ Department of Cardiology, 981 Hospital of Joint Logistics Support Force, Chengde, Hebei 067000, P. R. China.

${ }^{2}$ Department of Cardiology, Xijing Hospital, Airforce Military Medical University, Xi'an, Shaanxi 710032, P. R. China.

${ }^{3}$ Department of Health Statistics, Airforce Military Medical University, Xi'an, Shaanxi 710032, P. R. China.

${ }^{4}$ Department of Cardiology, The First Affiliated Hospital of Xi'an Medical University, Xi'an, Shannxi 710077, P. R. China.

${ }^{5}$ Department of Cardiology, No.971 Hospital of the PLA Navy, Qingdao, Shandong 266071, P. R. China.

${ }^{6}$ Cadet Brigade, School of Basic Medicine, Airforce Military Medical University, Xi'an, Shannxi 710032, P.R. China. 
${ }^{7}$ Department of Naval Medicine, Naval Medical University, Shanghai 200433, P. R. China

${ }^{8}$ Department of Pharmacogenomics, Airforce Military Medical University, Xi'an, Shaanxi 710032, P. R. China.

${ }^{9}$ Department of Cardiology, Hanyin County People's Hospital, Ankang, Shaanxi 725100, P. R. China

\section{References}

1. Fefer $P$, Knudtson ML, Cheema AN, et al. Current perspectives on coronary chronic total occlusions: the Canadian Multicenter Chronic Total Occlusions Registry. J Am Coll Cardiol. 2012;59(11):991997.

2. Galassi AR, Werner GS, Boukhris M, et al. Percutaneous recanalisation of chronic total occlusions: 2019 consensus document from the EuroCTO Club. Eurolntervention. 2019;15(2):198-208.

3. Neumann FJ, Sousa-Uva M, Ahlsson A, et al. 2018 ESC/EACTS Guidelines on myocardial revascularization. Eur Heart J. 2019;40(2):87-165.

4. Christakopoulos GE, Christopoulos G, Carlino M, et al. Meta-analysis of clinical outcomes of patients who underwent percutaneous coronary interventions for chronic total occlusions. Am J Cardiol. 2015;115(10):1367-1375.

5. Valgimigli M, Bueno H, Byrne RA, et al. 2017 ESC focused update on dual antiplatelet therapy in coronary artery disease developed in collaboration with EACTS: The Task Force for dual antiplatelet therapy in coronary artery disease of the European Society of Cardiology (ESC) and of the European Association for Cardio-Thoracic Surgery (EACTS). Eur Heart J, 2018, 39(3): 213-260.

6. Yong Huo, Young-Hoon Jeong, Yanjun Gong, et al. 2018 update of expert consensus statement on antiplatelet therapy in East Asian patients with ACS or undergoing PCI. Science Bulletin. 2019; 64: 166-179.

7. 2012 Writing Committee Members, Jneid H, Anderson JL, et al. 2012 ACCF/AHA focused update of the guideline for the management of patients with unstable angina/Non-ST-elevation myocardial infarction (updating the 2007 guideline and replacing the 2011 focused update): a report of the American College of Cardiology Foundation/American Heart Association Task Force on practice guidelines. Circulation, 2012. 126(7): 875-910.

8. Wang Y, Zhao HW, Wang CF, et al. Efficacy and safety of standard and low dose Ticagrelor versus Clopidogrel in east Asian Patients with chronic total occlusion undergoing percutaneous coronary intervention: a single center retrospective study. BMC Cardiovasc Disord. 2020;20(1):109.

9. Hicks KA, Mahaffey KW, Mehran R, et al. 2017 Cardiovascular and Stroke Endpoint Definitions for Clinical Trials. J Am Coll Cardiol, 2018. 71(9): 1021-1034.

10. Thygesen K, Alpert JS, Jaffe AS, et al. Fourth universal definition of myocardial infarction guidelines. Eur Heart J 2018; 40: 237-69. 
11. Scheinert D, Micari A, Brodmann M, et al. Drug-Coated Balloon Treatment for Femoropopliteal Artery Disease. Circ Cardiovasc Interv, 2018. 11(10): e005654.

12. Mehran R, Rao SV, Bhatt DL, et al. Standardized bleeding definitions for cardiovascular clinical trials: a consensus report from the Bleeding Academic Research Consortium. Circulation, 2011. 123(23): 2736-47.

13. Choi JH, Seo JM, Lee DH, Park K, Kim YD. Clinical utility of new bleeding criteria: a prospective study of evaluation for the Bleeding Academic Research Consortium definition of bleeding in patients undergoing percutaneous coronary intervention. J Cardiol. 2015;65(4):324-329.

14. Lee SW, Lee PH, Ahn JM, et al. Randomized Trial Evaluating Percutaneous Coronary Intervention for the Treatment of Chronic Total Occlusion. Circulation. 2019;139(14):1674-1683.

15. Costa F, Van Klaveren D, Feres F, et al. Dual Antiplatelet Therapy Duration Based on Ischemic and Bleeding Risks After Coronary Stenting. J Am Coll Cardiol. 2019;73(7):741-754.

16. Sachdeva A, Hung YY, Solomon MD, McNulty EJ. Duration of Dual Antiplatelet Therapy After Percutaneous Coronary Intervention for Chronic Total Occlusion. Am J Cardiol. 2020;132:44-51.

17. Levine GN, Bates ER, Bittl JA, et al. 2016 ACC/AHA guideline focused update on duration of dual antiplatelet therapy in patients with coronary artery disease: A report of the American College of Cardiology/American Heart Association Task Force on Clinical Practice Guidelines. J Thorac Cardiovasc Surg, 2016. 152(5): 1243-1275.

18. Husted S, James S, Becker RC, et al. Ticagrelor versus Clopidogrel in elderly patients with acute coronary syndromes: a substudy from the prospective randomized PLATelet inhibition and patient Outcomes (PLATO) trial. Circ Cardiovasc Qual Outcomes, 2012. 5(5): 680-8.

19. Kang HJ, Clare RM, Gao R, et al. Ticagrelor versus Clopidogrel in Asian patients with acute coronary syndrome: A retrospective analysis from the Platelet Inhibition and Patient Outcomes (PLATO) Trial. Am Heart J, 2015. 169(6): 899-905.e1.

20. Chen IC, Lee CH, Fang CC, et al. Efficacy and safety of Ticagrelor versus Clopidogrel in acute coronary syndrome in Taiwan: A multicenter retrospective pilot study. J Chin Med Assoc, 2016. 79(10): 521-30.

21. Silvain J, Lattuca B, Beygui F, et al. Ticagrelor versus Clopidogrel in elective percutaneous coronary intervention (ALPHEUS): a randomised, open-label, phase 3b trial. Lancet. 2020;396(10264):17371744.

22. You SC, Rho Y, Bikdeli B, et al. Association of Ticagrelor vs Clopidogrel With Net Adverse Clinical Events in Patients With Acute Coronary Syndrome Undergoing Percutaneous Coronary Intervention. JAMA. 2020;324(16):1640-1650.

23. Turgeon RD, Koshman SL, Youngson E, et al. Association of Ticagrelor vs Clopidogrel with Major Adverse Coronary Events in Patients with Acute Coronary Syndrome Undergoing Percutaneous Coronary Intervention. JAMA Intern Med. 2020;180(3):420-428.

24. Kang J, Kim HS. The Evolving Concept of Dual Antiplatelet Therapy after Percutaneous Coronary Intervention: Focus on Unique Feature of East Asian and "Asian Paradox". Korean Circ J. 
2018;48(7):537-551.

25. Goto S, Huang CH, Park SJ, Emanuelsson H, Kimura T. Ticagrelor vs. clopidogrel in Japanese, Korean and Taiwanese patients with acute coronary syndrome - randomized, double-blind, phase III PHILO study. Circ J. 2015;79(11):2452-2460.

26. Park DW, Kwon O, Jang JS, et al. Clinically Significant Bleeding With Ticagrelor Versus Clopidogrel in Korean Patients With Acute Coronary Syndromes Intended for Invasive Management: A Randomized Clinical Trial. Circulation. 2019;140(23):1865-1877.

27. Ahn JH, Ahn Y, Jeong MH, et al. Ticagrelor versus Clopidogrel in acute myocardial infarction patients with multivessel disease; From Korea Acute Myocardial Infarction Registry-National Institute of Health. J Cardiol. 2020;75(5):478-484.

28. Gimbel M, Qaderdan K, Willemsen L, et al. Clopidogrel versus Ticagrelor or prasugrel in patients aged 70 years or older with non-ST-elevation acute coronary syndrome (POPular AGE): the randomised, open-label, non-inferiority trial. Lancet. 2020;395(10233):1374-1381.

\section{Figures}




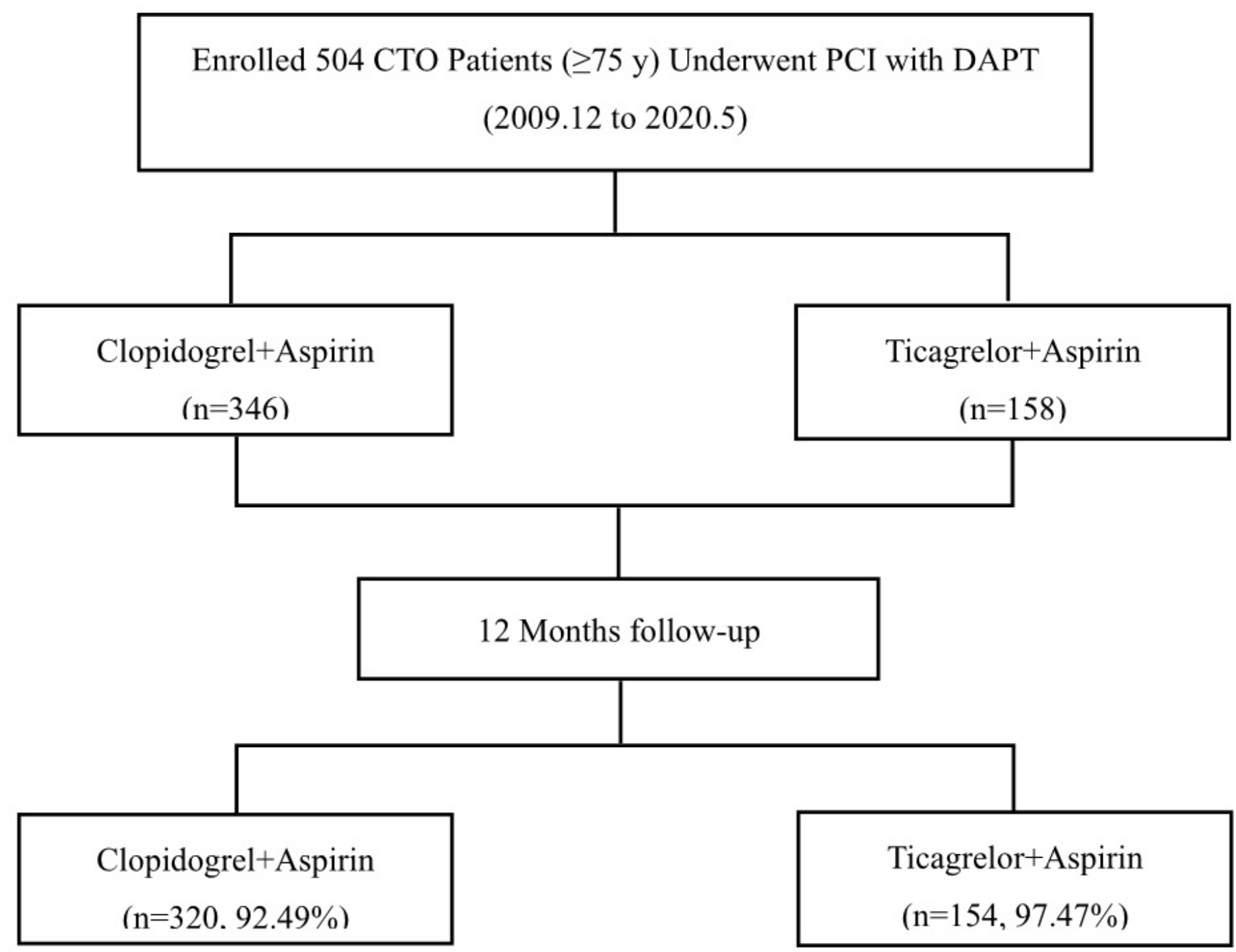

Figure 1

Study workflow 


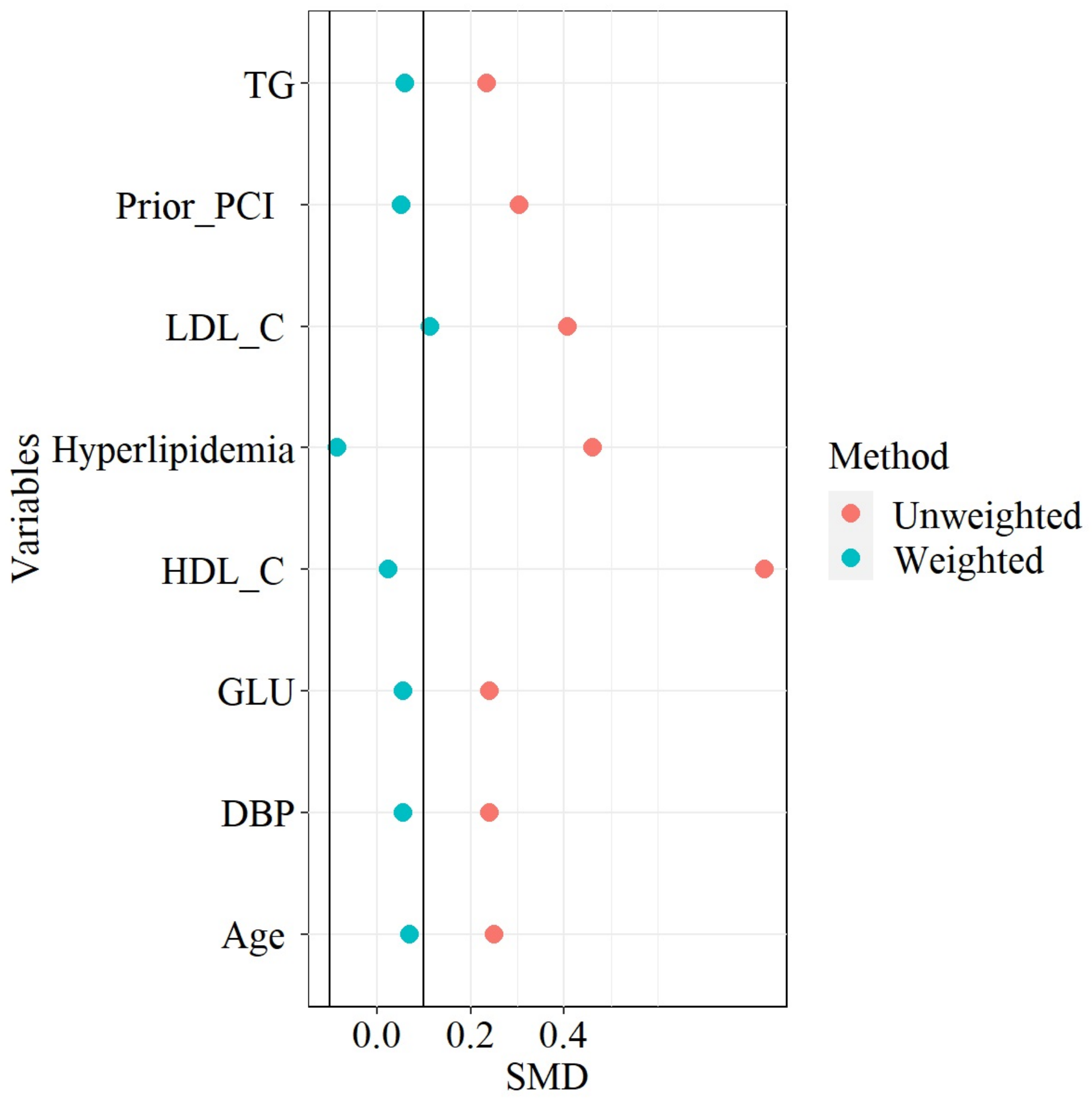

Figure 2

Standardized mean differences weighted and unweighted propensity score matching for the corresponding variable 


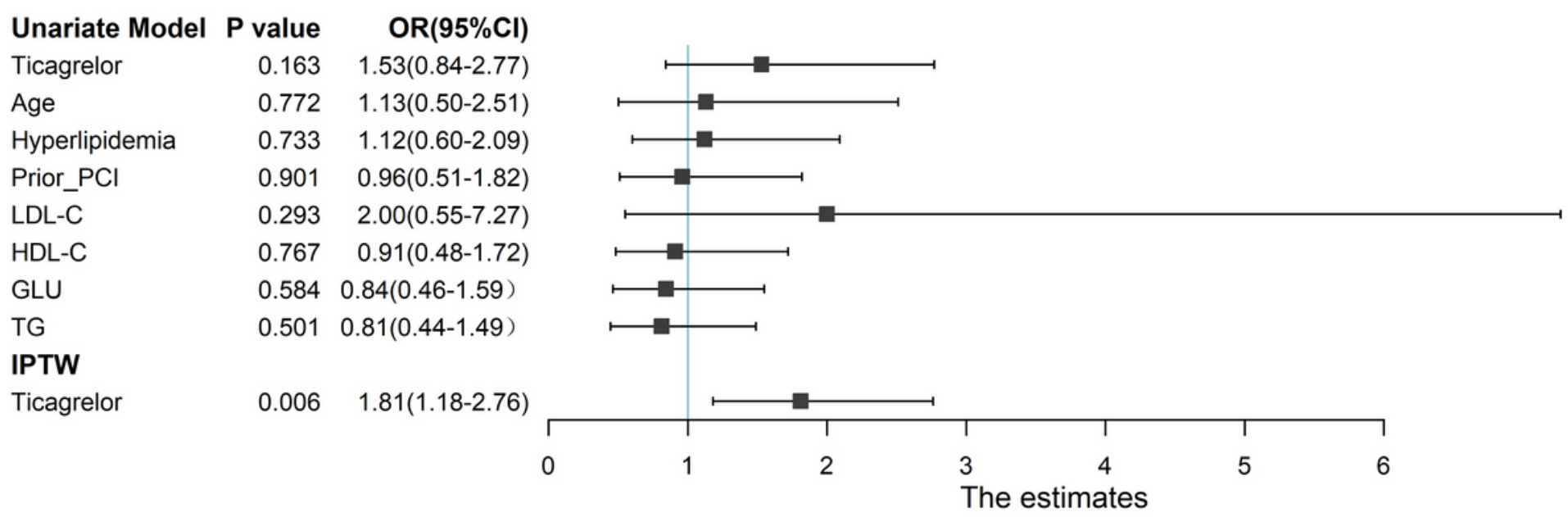

Figure 3

Unvariate analysis and IPTWs estimate of ticagrelor (vs. clopidogrel) on the occurrence of bleeding 